ORIGINAL ARTICLE

\title{
Computer based receptogram approach: an objective way of assessing immunohistochemistry of androgen receptor staining and its correlation with hormonal response in metastatic carcinoma of prostate
}

\author{
G Nabi, A Seth, A K Dinda, N P Gupta
}

J Clin Pathol 2004:57:146-150

See end of article for authors' affiliations

Correspondence to: Mr G Nabi, Department of Surgery, Medical School, University of Aberdeen, Polwarth Building Foresterhill, Aberdeen AB25 2ZD, UK; nabeegholam@hotmail. com

Accepted for publication 29 July 2003

\begin{abstract}
Aims: To categorise the immunostaining heterogeneity of androgen receptors in metastatic carcinoma of the prostate using a pattern oriented approach and to correlate the results with response to hormonal treatment.

Methods: Paraffin wax embedded tumour sections from 85 patients with metastatic carcinoma of the prostate were processed for immunocytochemistry and stained for the androgen receptor using antiandrogen receptor antibodies. A computer based image analysis system was used to analyse the pattern of nuclear immunostaining in a minimum of 500 nuclei/slide. Depending on the nuclear receptor content and concentration, receptogram patterns were established for each specimen. The receptogram pattern was correlated with clinical response to hormonal treatment.

Results: Clinical response to hormonal treatment was documented using prostate specific antigen as the marker into responders (good, fair, stable) and non-responders. Forty four of 48 patients who responded to hormonal treatment had type 1 (35) or type 3 (nine) receptograms, which are characterised by a unimodal peak or multimodal peaks within a narrow concentration range. Thirteen of the 18 patients who stabilised had type 1 or type 3 receptograms. Seventeen of the 19 patients who did not respond to hormonal treatment had either type 2 or type 4 receptograms, which are characterised by skewed or bimodal androgen receptor distribution. Positive and negative predictive values of receptograms were $96.5 \%$ and $63 \%$, respectively.

Conclusions: Image analysis of androgen receptor immunostaining with a receptogram oriented approach provides important prognostic information that can be used to predict response to hormone treatment in patients with metastatic carcinoma of the prostate.
\end{abstract}

\footnotetext{
C
} arcinoma of the prostate is the most common malignancy in men. ${ }^{1}$ Approximately $60 \%$ of newly diagnosed patients with prostate cancer already have metastatic disease for which hormonal manipulation remains the mainstay of treatment. The response to this form of treatment remains unpredictable and variable. ${ }^{23}$ Moreover, most patients eventually relapse to an androgen independent state and die. Presently, there is no useful method of predicting tumour behaviour and responsiveness to such treatment. Studies in the past have shown that mere positivity and negativity for androgen receptors of tumour cells may or may not predict response to hormonal treatment. ${ }^{4}$ On immunostaining, prostate carcinoma specimens show heterogeneity of androgen receptors in contrast to homogeneous staining of androgen receptors in normal prostate epithelium. ${ }^{5}$ Prins et al have shown that this heterogeneity is a useful indicator of tumour behaviour. ${ }^{6}$ Visual scoring of androgen receptor status of nuclei as positive or negative does not take into account the large variation in immunostaining intensity within a specimen or between specimens. Heterogeneity in the staining pattern also varies from one laboratory to another. To avoid this, an alternative and more objective method would be to use image analysis systems to measure androgen receptor staining intensities by a pattern oriented approach. The pattern oriented approach is a receptogram analysis based upon the quantification of the androgen receptor contents by immunocytochemical analysis. A receptogram is a composite of the univariate distribution of nuclear contents and their bivariate contour plots. ${ }^{78}$ Based on contour slopes, these are classified into subtypes and each is correlated with response to treatment. We present image analysis data from 85 androgen receptor positive, paraffin wax embedded prostate specimens of metastatic carcinoma of the prostate and correlate this with the response to hormonal treatment. In our present study, we have used a different antibody and image analysis system than reported previously. This system has been used for the expression of heat shock proteins in patients with end stage renal failure, as reported by us previously.

\begin{abstract}
"Visual scoring of androgen receptor status of nuclei as positive or negative does not take into account the large variation in immunostaining intensity within a specimen or between specimens"
\end{abstract}

\section{MATERIALS AND METHODS \\ Patients}

Between June 1995 and January 2001, 140 patients with metastatic carcinoma of the prostate were seen in the uromalignancy clinic of the All India Institute of Medical

Abbreviation: PSA, prostate specific antigen 
Sciences, New Delhi, India. Eighty five patients who met the criteria of sufficient archival paraffin wax embedded tumour specimen available for immunostaining and documented response to endocrine treatment were included in our study. Hormonal treatment consisted of either bilateral orchiedectomy (30 patients) or bilateral orchiedectomy with $250 \mathrm{mg}$ flutamide eight hourly ( 55 patients). There were no differences in terms of biochemical behaviour (increasing prostate specific antigen; PSA) or survival in one group compared with the other. The age of the patients ranged between 46 and 78 years (mean, 68). The clinical stage (Whitmore-Jewett) and Gleason score of disease were recorded at the start of hormonal treatment (table 1). The patients were followed three monthly, with symptoms and PSA concentrations being recorded.

The patients' response to hormonal treatment was classified as follows.

- Responders: patients who showed a biochemical response to hormonal manipulation and remained asymptomatic.

- Good: PSA less than $4 \mathrm{ng} / \mathrm{ml}$ for two or more years.

- Fair: PSA less than 4-10 ng/ml for two or more years.

- Stable: PSA decreased less than $50 \%$ but disease remained stable for two or more years.

- Non-responders: PSA continued to rise despite hormonal treatment and/or showed either progression of existing lesion or development of new lesions on bone scan.

Mean survival from the time of diagnosis was calculated.

\section{Androgen receptor immunostaining}

Formalin fixed, paraffin wax embedded sections were processed for immunocytochemistry using the microwave antigen retrieval method. The $4 \mu \mathrm{m}$ sections were dehydrated in graded ethanols, cleared in xylene, and mounted with Permount without being counterstained. The slides were stained with an antiandrogen antibody (monoclonal antiandrogen receptor antibody Ab-1; clone AR 441, Lab Vision, California, USA) (fig 1).

The immunostained slides were imaged using an image analysis system. ${ }^{3}$ The image analysis system consisted of a research microscope (B X 50; Olympus, Tokyo, Japan), 10 bit digital camera (Xilix Correco, Canada), image grabber card (F-64, Cerreco Corp, Quebec, Canada), and a personal computer (P-III; Digital Corp, California, USA). The image analysis software used was optimas 5.2 (Optimas Corp, California, USA). The integrated optical density and mean optical density of each nucleus were measured. Minimum numbers of 500 nuclei were analysed by the image analysis system. Nuclei were identified by the image analysis system automatically. The microscope imaging system was calibrated using the calibration dialogue program in the software and using a glass micrometer slide that had divisions in $\mu \mathrm{m}$ marked on it. Each lens was calibrated and these values were stored automatically.

Table 1 The stage of disease and Gleason score at the time of diagnosis and start of treatment for the 85 patients studied

\begin{tabular}{ll}
\hline Stage/score & N \\
\hline Stage & \\
D1 & 35 \\
D2 & 50 \\
Gleason score & \\
$<4$ & 16 \\
$4-7$ & 26 \\
$>7$ & 43 \\
\hline
\end{tabular}

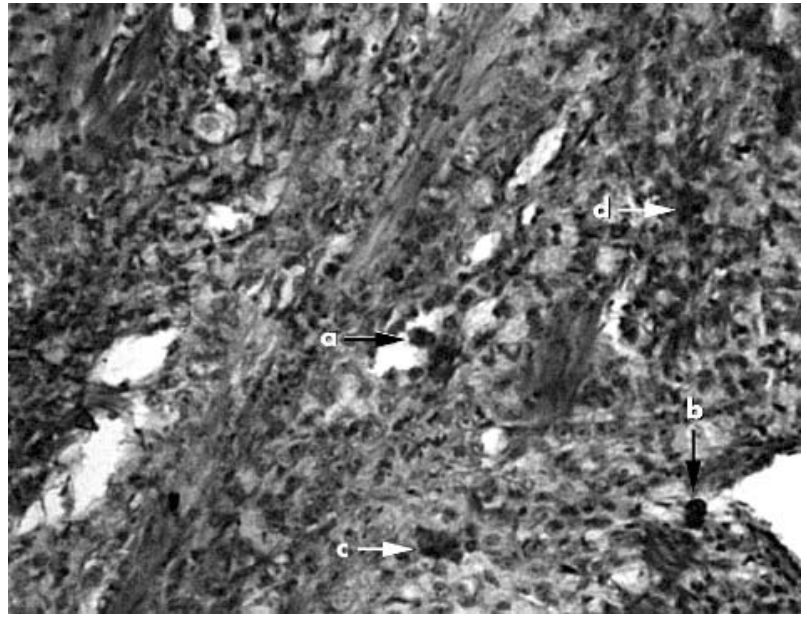

Figure 1 Photomicrograph showing immunostaining of androgen receptors ( $a-d$ ) using monoclonal antibody $A b-1$, clone AR 441; original magnification, $\times 220$.

The integrated optical density was plotted on the abscissa as a measure of the relative receptor content of individual nuclei and the percentage of nuclei expressing the different amounts of receptor were plotted on the ordinate. The receptograms were generated from the database and the patterns were analysed using standard software (Microsoft Excel and Access). ${ }^{9}$

\section{Receptogram classification and prediction of hormonal response}

Receptograms were classified into four possible types, depending on the androgen receptor immunostaining mean optical density distribution pattern, which is based upon the frequency distribution of the component positive or negative subpopulations of tumour cells, as described by Sklarew et al. ${ }^{8}$ The receptogram types are: type 1, unimodal androgen receptor positive distributions with a well defined peak (fig 2); type 2, a bimodal distribution, representing coexistent positive and negative androgen receptor subpopulation distributions (fig 3); type 3, multimodal androgen receptor positive distributions, with well defined peaks confined to less than a threefold concentration range (fig 4); and type 4, a highly skewed distribution, lacking well defined peaks and

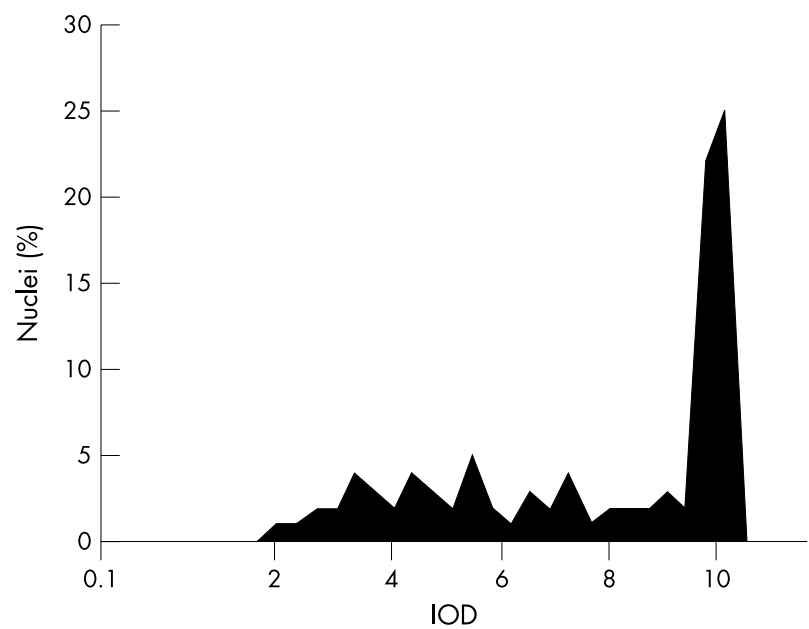

Figure 2 Type 1 receptogram distribution pattern of integrated optical density (IOD) of nuclei stained with an antiandrogen receptor antibody. 


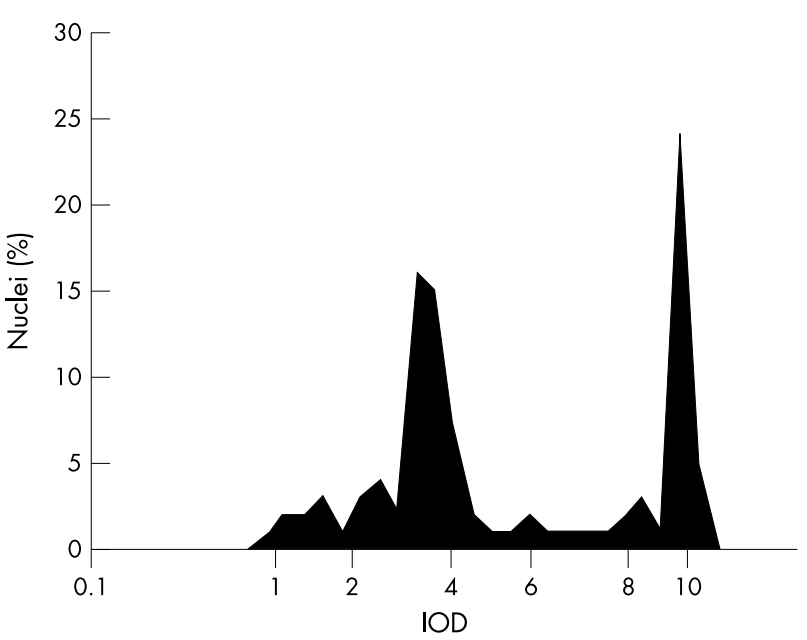

Figure 3 Type 2 receptogram distribution pattern of integrated optical density (IOD) of nuclei stained with an antiandrogen receptor antibody.

extending over a greater than threefold concentration range (fig 5).

\section{Statistical analysis}

Statistical analysis (the $\chi^{2}$ test) was carried out using SPSS7.5 software. A p value $<0.05$ was considered to be significant

\section{RESULTS}

The mean follow up was 34.6 months (range, 24-60). Of the 85 patients with prostatic tumours, 22 had a good response, 26 responded fairly, 18 stabilised on hormonal treatment, and in 19 patients the treatment failed. Table 2 details the receptogram patterns of each of the response groups. Nineteen of the 22 good responders had either type 1 or type 3 receptogram patterns. In contrast, 17 of the 19 patients who did not respond to hormonal treatment had either type 2 or type 4 receptogram patterns. The patients were divided into responders (good response, fair response, and stabilised) or non-responders (failed treatment) and the data were collated into $2 \times 2$ contingency tables for statistical analysis. The sensitivity and specificity with negative and positive predictive values were calculated.

The sensitivity of the test (the number of responders with either type 1 or type 3 receptograms) was $85 \%$, with a specificity of $89.5 \%$ (the number of failures with type 2 or 4 receptograms). The positive predictive and negative predictive values of this test were $96.6 \%$ and $63 \%$, respectively.

The diagnostic accuracy of this test was $85.9 \%$. When the responders were compared with the non-responders, the $\chi^{2}$ test with Yate's correction factor was 33.7 and p values were highly significant $(\mathrm{p}<0.0001)$. There was no significant correlation between Gleason grade and receptogram pattern on image analysis (table 3).

Table 2 Response to hormonal treatment in relation to receptogram pattern (types 1-4)

\begin{tabular}{lccccc}
\hline Response & N & Type 1 & Type 2 & Type 3 & Type 4 \\
\hline Responders & & & & & \\
$\quad$ Good & 22 & 15 & 2 & 5 & 1 \\
$\quad$ Fair & 26 & 20 & 1 & 4 & 1 \\
$\quad$ Stabilised & 18 & 10 & 2 & 3 & 3 \\
Non-responders & 19 & 1 & 7 & 1 & 10 \\
Total & 85 & 46 & 12 & 13 & 15 \\
\hline
\end{tabular}

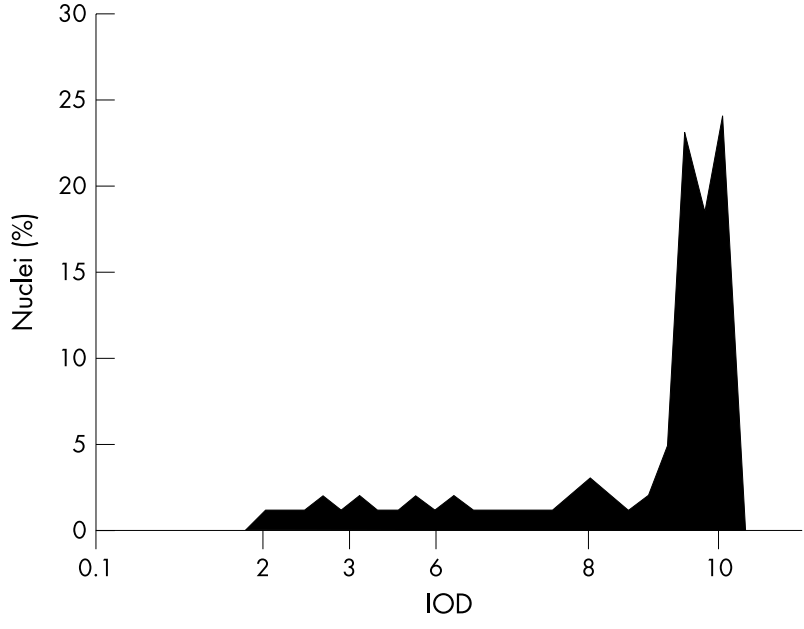

Figure 4 Type 3 receptogram distribution pattern of integrated optical density (IOD) of nuclei stained with an antiandrogen receptor antibody.

\section{DISCUSSION}

Carcinoma of the prostate is the second leading cause of death after lung cancer in men. ${ }^{1-7}$ Sixty per cent of patients present with metastatic disease at the time of diagnosis, and in such circumstances hormonal manipulation remains the only effective palliative measure. The relapse and progression of disease have been the main focus of attention since the introduction of endocrine treatment. Hormonal independence carries a poor prognosis and eventually results in death. The molecular basis of this phenomenon is poorly understood, although it is associated with greater genetic instability and increased risk of hormonal independence. ${ }^{10}$ To predict the response to hormonal treatment, a marker for genetic instability is required. Previous studies have established that mere androgen receptor positivity or negativity by itself does not accurately predict a positive or negative response to hormonal treatment.

It has been noted that the normal epithelium of the prostate shows a homogeneous immunostaining pattern. Genetic instability within the tumour cells results in the expression of heterogeneity: the greater the intratumorous heterogeneity, the greater the genetic instability. Thus, heterogeneity is a possible marker for genetic instability. In a previous evaluation, this phenomenon was reported to be useful in predicting response to hormonal treatment. ${ }^{11}$

The variability of androgen receptor staining among responders, non-responders, and stabilised patients can be

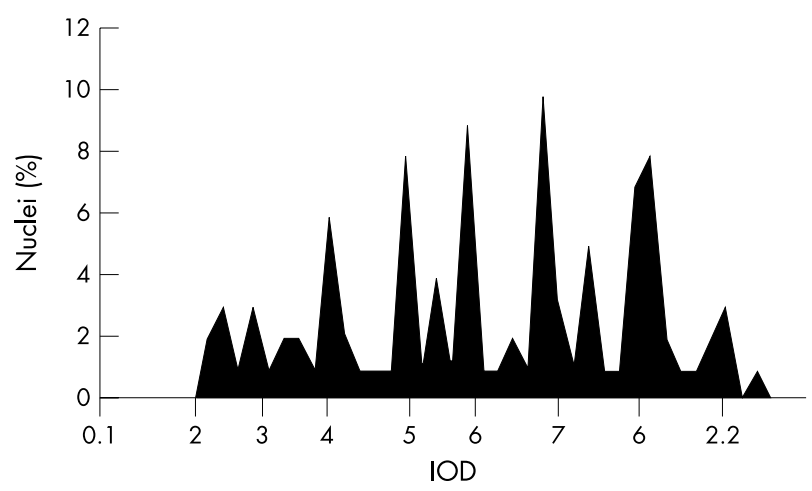

Figure 5 Type 4 receptogram distribution pattern of integrated optical density (IOD) of nuclei stained with an antiandrogen receptor antibody. 
Table 3 Correlation between Gleason score and receptogram results in relation to response to hormonal treatment

\begin{tabular}{lrrrrrr}
\hline \multirow{2}{*}{$\begin{array}{l}\text { Gleason } \\
\text { score }\end{array}$} & \multicolumn{9}{c}{ Type of receptogram } & \\
\cline { 2 - 5 } & $\mathbf{1}$ & $\mathbf{2}$ & \multicolumn{3}{c}{$\mathbf{3}$} & \multicolumn{4}{c}{$\mathbf{4}$} & Responders & Non-responders \\
\hline$<4$ & 4 & 6 & 3 & 3 & 7 & 9 \\
$4-7$ & 8 & 7 & 5 & 6 & 12 & 14 \\
$>7$ & 11 & 8 & 10 & 14 & 24 & 19 \\
\hline
\end{tabular}

seen using an unaided microscope, although the results are subjective and vary from one laboratory to another. Prins et al attempted to determine androgen receptor status by "histoscoring", ${ }^{12}$ which incorporates immunostaining intensity, and correlated it with the responsiveness of tumours to treatment. The positive and negative predictive values for endocrine response were $71 \%$ and $62 \%$, respectively. To improve accuracy and have a more objective predictor, immunostaining with image analysis and receptogram classification (pattern oriented approach) was evaluated for metastatic carcinoma of the prostate. In addition, the pattern oriented approach is independent of the differences in staining intensity caused by immunohistochemical techniques.

"Those who responded to hormonal treatment either had a well defined single peak (type 1) or multiple discrete peaks in a narrow range (type 3$)^{\prime \prime}$

Receptogram analysis was previously evaluated as a pattern oriented approach for endocrine response prediction in carcinoma of the breast and found to be a useful marker. ${ }^{8}$ In their preliminary observation, Pertschuk et al found a similar staining heterogeneity in carcinoma of the prostate. ${ }^{13}$ When an image analysis system was used, it was also noted that low numbers of androgen receptors were present within the tumour cells of patients who had been labelled as androgen receptor negative by visual determination.

In our present study, we carried out immunostaining and receptogram analysis in prostate tumour specimens obtained from 85 patients after they had undergone radical prostatectomy or tru-cut biopsy, but before they began hormonal treatment. Image analysis produced four types of receptogram. Type 2 and 4 receptograms were representative of a considerable amount of heterogeneity within the tumour, probably as a result of the presence of multiple tumour proliferating subpopulations with a higher genetic instability than the cells characterised by type 1 and 3 receptograms. Those who failed to respond to hormonal treatment in our present study had either coexistent androgen receptor positive and negative subpopulations within the same tumour cells (type 2) or a highly skewed mosaic staining distribution lacking well defined peaks (type 4). In contrast, those who responded to hormonal treatment either had a well defined single peak (type 1) or multiple discrete peaks in a narrow range (type 3 ). We found that image analysis can discriminate between those who show a good or a poor response to hormonal treatment, as has been noted previously. ${ }^{14}{ }^{15}$ Our study used a different image analysis system and a different source of antiandrogen antibody, highlighting the fact that limited resources of certain antibodies or the use of different image analysis systems should not affect the applicability of androgen receptor image analysis to the analysis of metastatic carcinoma of the prostate. We found no

\section{Take home messages}

- Computer based image analysis of androgen receptors can provide useful information in patients with metastatic carcinoma of the prostate with regard to response to hormonal treatment

- The positive predictive and negative predictive values of this test were $96.6 \%$ and $63 \%$, respectively, and the diagnostic accuracy was $85.9 \%$

- This method can objectively assess androgen receptor status and provide information regarding tumour biology

- There was no significant correlation between Gleason grade and receptogram pattern

- Further studies are needed before useful conclusions can be drawn

significant correlation between Gleason grade and receptogram pattern. It is our belief that this phenomenon requires further study, before useful conclusions can be drawn.

\section{CONCLUSION}

Computer based image analysis of androgen receptors provides useful prognostic information in patients with metastatic carcinoma of the prostate who are on hormonal treatment. It is an objective assessment of androgen receptor status that can provide information regarding tumour biology.

\section{ACKNOWLEDGEMENTS}

This work has been possible through an intramural faculty grant by AIIMS, New Delhi, India. We thank Dr M A Choudhary of the Department of Molecular and Cell Biology, University of Aberdeen for useful suggestions and review.

\section{Authors' affiliations}

G Nabi, Department of Surgery, Medical School, University of Aberdeen, Aberdeen AB25 2ZD, UK

A Seth, Department of Urology, All India Institute of Medical Sciences, New Delhi-29, India

A K Dinda, Department of Pathology, All India Institute of Medical Sciences

N P Gupta, Departments of Urology and Pathology, All India Institute of Medical Sciences

This work was presented at the 18th European Association of Urologists' annual meeting on 12-15 March 2003, Madrid, Spain and the Urological Society of India, annual meeting 11-13 October 2001, Udiapur, India.

\section{REFERENCES}

1 Catalona WJ, Scott WW. Carcinoma of the prostate. In: Walsh PC, Retik AA, Vaughan ED, et al, eds. Campbell's urology, Vol. 2. Philadelphia: WB Saunders Co, 1986:1463-534.

2 Sadi MV, Walsh PC, Barrack ER. Immunohistochemical study of androgen receptors in metastatic prostate cancer: comparison of receptor content and response to hormonal therapy. Cancer 1991;67:3057-64.

3 Sadi $M$, Barrack E. Image analysis of androgen receptor immunostaining in metastatic carcinoma of the prostate. Cancer 1993;71:2574-6.

4 Barrack ER, Tindall DJ. A critical evaluation of the use of androgen receptor assay to predict the androgen responsiveness of prostate cancer. Prog Clin Biol Res 1987;239:155-87.

5 Brolin J, Skoog L, Ekman P. Immunohistochemistry and biochemistry in detection of androgen, progesterone and estrogen receptors in benign and malignant human prostate tissue. Prostate 1992;20:281-5.

6 Prins GS, Birch L, Greece GL. Androgen receptor localization in different cell types of the adult rat prostate. Endocrinology 1991;129:3187-9. 
7 Pertschuk LP, Feldman JG, Kim YD, et al Estrogen receptor immunocytochemistry in paraffin embedded tissues with ER1D5 predicts breast cancer endocrine response more accurately than H222Spgamma in frozen sections or cytosol-based ligand binding assays. Cancer 1996:77:2514-18.

8 Sklarew RJ, Pertschuk LP. Quantitation of the immunocytochemical assay for estrogen receptor proteins (ER. ICA) in human breast cancer by television imaging. J Histochem Cytochem 1991;35:1253-5.

9 Dinda AK, Mathur M, Guleria S, et al. Heat shock protein (HSP) expression and proliferation of tubular cells in end stage renal disease with and without hemodialysis. Nephrol Dial Transplant 1998;13:99-105.

10 Isaac JT, Wake N, Coffey DS, et al. Genetic instability coupled to clonal selection as a mechanism for tumour progression in the Dunning R-3327 rat prostatic adenocarcinoma system. Cancer Res 1982;42:2353-61.
11 Sadi M, Walsh P, Barrack E. Immunohistochemical study of androgen receptor in metastatic cancer. Cancer 1994;67:3057-62.

12 Prins GS, Robert JS, Pertschuk LP. Image analysis of androgen receptors immunostaining in prostate cancer accurately predicts response to hormonal treatment. J Urol 1998:159:641-9.

13 Pertschuk LP, Schaeffer H, Feldman JG, et al. Immunostaining for prostate cancer androgen receptor in paraffin identifies a subset of men with a poor prognosis. Lab Invest 1995;73:302.

14 Sklarew R, Bodner S, Pertschuk L. Comparison of microscopic imaging strategies for evaluating immunocytochemical (PAP) steroid receptors heterogeneity. Cytometry 1991;12:207-9.

15 Tilley W, Lim-Tio S, Horsfall DJ, et al. Detection of discrete androgen receptor epitopes in prostate cancer by immunostaining: measurement by color video image analysis. Cancer Res 1994;54:4096-8.

\section{Clinical Evidence - Call for contributors}

Clinical Evidence is a regularly updated evidence based journal available worldwide both as a paper version and on the internet. Clinical Evidence needs to recruit a number of new contributors. Contributors are health care professionals or epidemiologists with experience in evidence based medicine and the ability to write in a concise and structured way.

\section{Currently, we are interested in finding contributors with an interest in} the following clinical areas:

Altitude sickness; Autism; Basal cell carcinoma; Breast feeding; Carbon monoxide poisoning; Cervical cancer; Cystic fibrosis; Ectopic pregnancy; Grief/bereavement; Halitosis; Hodgkins disease; Infectious mononucleosis (glandular fever); Kidney stones; Malignant melanoma (metastatic); Mesothelioma; Myeloma; Ovarian cyst; Pancreatitis (acute); Pancreatitis (chronic); Polymyalgia rheumatica; Post-partum haemorrhage; Pulmonary embolism; Recurrent miscarriage; Repetitive strain injury; Scoliosis; Seasonal affective disorder; Squint; Systemic lupus erythematosus; Testicular cancer; Varicocele; Viral meningitis; Vitiligo However, we are always looking for others, so do not let this list discourage you.

Being a contributor involves:

- Appraising the results of literature searches (performed by our Information Specialists) to identify high quality evidence for inclusion in the journal.

- Writing to a highly structured template (about 2000-3000 words), using evidence from selected studies, within 6-8 weeks of receiving the literature search results.

- Working with Clinical Evidence Editors to ensure that the text meets rigorous epidemiological and style standards.

- Updating the text every eight months to incorporate new evidence.

- Expanding the topic to include new questions once every 12-18 months.

If you would like to become a contributor for Clinical Evidence or require more information about what this involves please send your contact details and a copy of your CV, clearly stating the clinical area you are interested in, to Claire Folkes (cfolkes@bmigroup.com).

\section{Call for peer reviewers}

Clinical Evidence also needs to recruit a number of new peer reviewers specifically with an interest in the clinical areas stated above, and also others related to general practice. Peer reviewers are health care professionals or epidemiologists with experience in evidence based medicine. As a peer reviewer you would be asked for your views on the clinical relevance, validity, and accessibility of specific topics within the journal, and their usefulness to the intended audience (international generalists and health care professionals, possibly with limited statistical knowledge). Topics are usually 2000-3000 words in length and we would ask you to review between 2-5 topics per year. The peer review process takes place throughout the year, and our turnaround time for each review is ideally 10-14 days.

If you are interested in becoming a peer reviewer for Clinical Evidence, please complete the peer review questionnaire at www.clinicalevidence.com or contact Claire Folkes(cfolkes@bmigroup.com). 